\title{
Proyectando una sociología latinoamericana: el Boletín del Instituto de Sociología de la Universidad de Buenos Aires y Francisco Ayala
}

\author{
Luis A. Escobar ${ }^{1}$
}

\author{
Fecha de recepción: 19 de junio de 2017
}

Fecha de aprobación: 2 de agosto de 2017

\begin{abstract}
Resumen
El presente artículo plantea recuperar la experiencia del Instituto de Sociología de la Universidad de Buenos Aires y su órgano de publicación, el Boletín del Instituto de Sociología, como punto de apertura a ciertas configuraciones regionales que se direccionaron en la búsqueda de innovaciones en el campo de la sociología.

Esta exploración propone re-articular algunos trayectos de una historia social de la sociología latinoamericana en la década del cuarenta del siglo XX y para ello focaliza en las intervenciones, vínculos, búsquedas y propuestas del español Francisco Ayala en el Boletín, puesto que es uno de los referentes en la conformación de un diálogo regional.
\end{abstract}

Palabras clave: sociología latinoamericana, renovación disciplinar, diálogos regionales, tradiciones sociológicas, estatuto científico, Ayala.

\section{Projecting a latin american sociology: the Bulletin of the Institute of Sociology of the University of Buenos Aires and Francisco Ayala}

\begin{abstract}
This article aims to recover the experience of the Institute of Sociology of the University of Buenos Aires and its publication body, the Bulletin of the Institute of Sociology as an opening to certain regional configurations directed in the search for innovations in the sociology field.

This exploration proposes to re-articulate some trajectories of a social history of Latin American sociology in 1940's and to that end it focuses on interventions, links, searches and proposals of the Spaniard Francisco Ayala in the Newspaper, since he is one of the referents in the formation of a regional dialogue.
\end{abstract}

1 Dr. (c) en Estudios Sociales de América Latina, Universidad de Córdoba. Investigador y colaborador en el CAI+D "Política y Cultura" de la Universidad Nacional del Litoral (Santa Fe, Argentina). Contacto: 1.al.escobar@gmail.com. 
Key words: Latin American sociology, disciplinary renewal, regional dialogues, sociological traditions, scientific status, Ayala.

\title{
Projetando uma sociologia latino-americana: o Boletim do Instituto de Sociologia da Universidade de Buenos Aires e Francisco Ayala
}

\begin{abstract}
Resumo
O presente artigo planteia recuperar a experiência do Instituto de Sociologia da Universidade de Buenos Aires e seu órgão de publicação, o Boletim do Instituto de Sociologia, como ponto de abertura para certas configurações regionais que foram direcionadas para a busca de inovações no campo de sociologia.

Esta exploração propõe a rearticular algumas trajetórias de uma história social da sociologia latino-americana na década do 40 do século XX e para isso se concentra nas intervenções, vínculos, buscas e propostas do espanhol Francisco Ayala no Boletim, já que é um dos referentes para a conformação de um diálogo regional.
\end{abstract}

Palavras-chave: sociologia latino-americana, renovação disciplinar, diálogos regionais, tradições sociológicas, código científico, Ayala.

\section{Introducción: la traza de una sociología}

La sociología en Latinoamérica cuenta con extensos e intensos recorridos sobre los cuales se ha asentado. Para comprender e interpretar estas densidades desde un presente situado en herencias y problemáticas, acaso resulte preciso desenhebrar algunas puntas de acceso a hilos de continuidades de difícil observación desde las superficies de la madeja. En virtud de eso procuraremos recuperar algunas de las luchas por la imposición de definiciones y búsquedas de institucionalización, de sustentos disciplinares propios, para desde allí re-articular trayectos de una historia social de la sociología latinoamericana. Consideramos que este ejercicio puede devolvernos imágenes en las cuales los horizontes epócales de la disciplina se comprendan desde una mayor complejidad, en relación y en contexto, resituando debates, perspectivas, exploraciones, prácticas e incluso clásicos no convencionales y/o quizás olvidados.

En particular, la década del cuarenta del siglo pasado se constituye en el escenario de proyectos y propuestas de estudios so- 
ciológicos en abierta modernización, articulándose grupos que promueven vínculos, fundan afinidades y conexiones, en busca de arraigar una renovación sociológica regional.

Como presentan algunas investigaciones en la región Argentina, en la década del cuarenta a través de experiencias de gradual institucionalización se vislumbran indicadores de una reorganización de la sociología (González Bollo, 1999; Blanco, 2006; Pereyra, 2007). Las primeras investigaciones que Alfredo Poviña comienza a publicar en la revista Cursos y Conferencias (órgano oficial de publicación del Colegio Libre de Estudios Superiores de Buenos Aires) tratan de indagaciones sobre el estado de la sociología en Argentina; para lo cual indaga información y antecedentes de las cátedras universitarias argentinas. Poviña, en base a las mismas, argumenta que se puede hablar de un "movimiento sociológico" en la Argentina, dado que, aunque desvinculadas entre sí, las cátedras de sociología presentan coincidencias en sus líneas generales (Escobar, 2016). Este trabajo es continuado y ampliado por Poviña, quien en 1941 publica un libro en el Fondo de Cultura Económica de México, bajo el título Historia de la Sociología en Latinoamérica. Estos diagnósticos epocales de Poviña son antecedentes para observar el progresivo interés consciente de necesidades vinculares y programáticas de una sociología que comenzaba a descubrirse y relacionarse regionalmente, a pesar de las diferencias y particularidades.

En este marco, el Boletín del Instituto de Sociología de la Universidad de Buenos Aires (UBA) aporta una sugestiva información que merece en sí misma una investigación. En cierta manera esta publicación da cuenta del proceso de renovación disciplinar, de los diálogos regionales y el conjunto de acciones y estrategias que se comienzan a articular y poner en juego. Debido a nuestro recorte $^{2}$ puntuaremos el análisis en las intervenciones de la sec-

2 Es importante aclarar que este artículo se enmarca en un trabajo de tesis doctoral en curso, por lo cual los recortes, propuestas y análisis están en directa relación con el mencionado marco. 
ción "Información General", y, en particular, en la sub-sección “Notas sobre la enseñanza de la sociología en América y la Argentina", para luego focalizar fundamentalmente en los escritos, búsquedas y propuestas del español Francisco Ayala (Granada, 1906 - Madrid, 2009)3 , como uno de los referentes dentro de la construcción de un diálogo regional en la renovación de la disciplina sociológica.

\section{La experiencia del Instituto de Sociología de la UBA y su órgano de publicación}

En 1940, en la Facultad de Filosofía y Letras (FFyL) de la UBA, se funda el Instituto de Sociología, presidido y constituido por Ricardo Levene (1885-1959), a esa altura ya un reconocido historiador argentino de importante trayectoria y referente de la Nueva Escuela Histórica, pero también jurista y sociólogo. Levene había desarrollado actividades catedráticas al frente de Sociología en la FFyL desde 1911, como suplente, y desde 1922 como titular, luego de la renuncia de Ernesto Quesada. Dichas actividades se vincularon con una larga trayectoria del mencionado, ya que, a modo de ejemplo, su tesis doctoral, defendida en 1907 en la misma UBA para acceder al grado de doctor en Jurisprudencia y Leyes, fue un compendio de las principales teorías sociológicas de su tiempo (González Bollo, 1999, p. 22; Pereyra, 2007, pp. 155-156; Blanco, 2006, pp. 52-58; Blanco y Jackson, 2015, p. 106; Devoto y Pagano, 2009).

3 Doctor en Derecho en la Universidad de Madrid (1929), estudió Filosofía y Letras, en Madrid, y Filosofía Política y Sociología General, en Alemania, entre 1929-31. Desde 1933 hasta 1936 enseñó como catedrático de Sociología y Ciencias Políticas en la Universidad de Madrid. En 1932 fue nombrado Letrado Asesor de la Cámara de Diputados de Madrid; en 1934 se desempeñó como titular de Derecho Político en la Universidad de Lagunas. El inicio de la Guerra Civil lo sorprende dando conferencias en Latinoamérica; cuando retorna a España ejerce funciones en el Ministerio de Estado. Al caer la República se exilia en Argentina, donde permanece diez años (1939-1949), para luego trasladarse a Puerto Rico y finalizar su exilio en Estados Unidos (1976). Tras su exilio norteamericano regresa a España, con la reinstauración de la democracia, y reside en Madrid hasta su fallecimiento. 
Si bien el Instituto al que nos referimos tenía una existencia previa, dado que se había creado mediante una resolución en 1927 bajo el nombre de "Instituto de Sociología Argentina", con Levene no solo se modifica su nombre sino que se reorganiza. Estos espacios fueron impulsados, en gran medida, por la Reforma Universitaria de 1918 (Buchbinder, 2007, pp. 126-127) y constituyeron instancias que trataron de relacionar ciencia e investigación, es decir, instituir a la universidad como centro aglutinante y promotor de investigación, a la par que formador, aunque con escasas herramientas institucionales reales, que con el devenir del tiempo se fueron perfeccionando. En este contexto emergieron los primeros institutos de investigación en las universidades argentinas (Pereyra, 2007; Rawicz, 2012; Morales Martín, 2014).

En el cambio de nombre - de Instituto de Sociología Argentina por Instituto de Sociología - se observa un viraje producto quizás de los objetivos perseguidos por Levene y/o también de la comprensión y traducción de los cambios producidos en el contexto regional y mundial. Como sostenía Alfredo Poviña —quien participaría como "adscrito" en el Instituto- "la sociología nacional está condicionada previamente por la sociología general (...)" (1943, p. 160).

El proyecto encabezado por Levene sumó un conjunto de nombres vinculados a la sociología en cátedras tanto de Argentina como de Latinoamérica, en calidad de "adscritos" al Instituto. El elenco resultante de dichas invitaciones estuvo conformado, en el ámbito nacional, por Alfredo Poviña, Francisco Ayala, Raúl Orgaz, Alberto Baldrich, Gino Germani, Renato Treves y Jordán Bruno Genta. Mientras que del exterior se sumó Gilberto Freyre y Antonio Carneiro Leâo, de Brasil; Lucio Mendieta y Núñez y José Medina Echavarría, de México; Roberto Mac-Lean y Estenós, de Perú; Germán Arciniegas, de Colombia; y Justo Prieto, de Paraguay (Levene, 1947, p. 130).

Estas acciones institucionales muestran las intenciones vinculantes y de apertura en la nueva etapa abierta en 1940. Levene 
pretendía, por un lado, nuclear todas las cátedras universitarias de sociología del país para impulsar intercambios e investigaciones, con los objetivos de coordinar la enseñanza con la investigación y asesorar al Estado en materias sociales; por otro lado, buscaba contribuir a la creación de un Instituto Internacional de Sociología en América, acción para la cual incluía en el diálogo, la participación y las relaciones de los adscritos latinoamericanos, entre otras estrategias (Pereyra, 2007, p. 157).

Los objetivos propuestos para reorganizar el Instituto implicaron además la creación de áreas internas, que contenían recolección de datos y preocupaciones teóricas con el fin de analizar la "morfología social" del país y describir los fenómenos sociales a partir de datos estadísticos relevados de acuerdo con la orientación metodológica de la "Sociografía" (Levene, 1947, p. 128). Asimismo, se organizaron conferencias y reuniones de discusión e investigación, y entre 1942-1947 (primera época) se editaron desde el Instituto cinco números de un órgano de publicaciones propio denominado Boletín del Instituto de Sociología, la primera revista de sociología de Argentina; luego sus ediciones se interrumpirían hasta ser retomadas en 1952. Luego, para esta segunda época, tanto el Instituto como el Boletín ya no estaban a cargo de Levene y de sus principales colaboradores (Neiburg, 1998, pp. 186-188).

El primer número editado por el Boletín en mayo de 1942, está compuesto por un Índice subdivido en seis partes. En la primera se encuentra una presentación de la publicación y artículos varios, uno de ellos tiene por autor a Ricardo Levene y otro a Emilio Ravignani - colega de Levene y decano de la FFyL, encargado de su designación al frente del Instituto-. Ambos exponen las características, organización y objetivos del Instituto de Sociología. Complementan el cuerpo central una serie de artículos con una interesante lista de autores y abordajes temáticos, que muestran de manera acorde los objetivos de apertura sostenidos por el Instituto:

- Gilberto Freyre, Factores sociales en la formación de la sociología brasileña. 
- Renato Treves, El problema de la sociedad en el pensamiento contemporáneo.

- Alfredo Poviña, La sociología en las universidades americanas.

- William Rex Crawford, Las escuelas de la sociología en Norteamérica.

- Rodolfo Mondolfo, Espíritu revolucionario y conciencia histórica.

- Alberto Baldrich, Libertad y determinismo en la sociología de Max Scheler.

- Francisco Ayala, Sociología: teoría y técnica.

- Gino Germani, La clase media en la ciudad de Buenos Aires. Estudio preliminar.

- Agustín V. Podestá, Acerca del Instituto de la opinión pública.

- Juan B. Molinari, La actualidad del problema sociológico y la idea de progreso social (A propósito de la traducción de trabajos científicos de C. Bouglé).

La composición del Boletín continúa con cinco secciones: "Galería de sociólogos americanos", "Datos sobre la realidad social argentina contemporánea”, "Noticias bibliográficas", "Comentarios e Información general".

\section{América y Argentina en el Boletín del Instituto de Sociología}

La Información general, que cierra el Índice del órgano de publicaciones, se inicia con un informe que se llama "Notas sobre la enseñanza de la sociología en América y la Argentina". Éste, a su vez, se divide en dos segmentos: "América del Sur" y "Argentina". El primero está a cargo del mexicano Carlos Echánove Trujillo, profesor de Sociología General en la Facultad de Derecho y Ciencias Sociales de la Universidad Nacional de México. Este escueto informe funciona como apertura de un panorama regional de la "observación" (obras y autores pioneros) y la enseñanza de la sociología (Echánove Trujillo, 1942, pp. 249-256). 
En todo caso se trata de una presentación resumida de algunos "precursores" regionales, así como una sucinta fundamentación de que la sociología en la región latinoamericana no solo se trata de una "materia de enseñanza" sino que además en los "programas de enseñanza figura a menudo un capítulo dedicado a la sociología vernácula" (Echánove Trujillo, 1942, p. 251). En relación con esta categoría, el autor parece referirse a estudios abocados a la historia y desarrollo de cada caso regional, con predominio de una mirada jurídica e institucional, aunque en algunos casos, como el ecuatoriano, aparecen algunas perspectivas de abordaje más relacionado a lo social.

Echánove Trujillo muestra la presencia de una "sociología vernácula" mediante citas de los programas de cátedra de la Universidad de Guayaquil (Ecuador), de la Universidad Central de Venezuela, de las universidades de Buenos Aires, Córdoba y Tucumán de Argentina ${ }^{4}$, del Colegio Universitario de Río de Janeiro (Brasil), de la Universidad de Chile y la Universidad Nacional de Colombia. En oposición a lo que ocurre en la Universidad de San Marcos en Perú y en la Facultad de Derecho y Ciencias Sociales de Montevideo (Uruguay), donde no se abordan temas desde esta categorización, según la lectura del autor.

Por otra parte, el segundo segmento del informe se presenta subdividido en localidades y autores de la siguiente manera:

- A) Córdoba, por Alfredo Poviña.

- B) Paraná, por Jordán Bruno Genta.

- C) Rosario, por Alberto Baldrich.

- D) Santa Fe, por Francisco Ayala.

- E) Tucumán, por Renato Treves.

Lo primero que llama la atención, a simple vista, es que no aparezcan identificados los casos de Buenos Aires y La Plata,

4 El caso argentino es el que el autor más atiende, incluso arriesga una conclusión: en la "sociología vernácula" local sus temas se "concretan más bien a la historia y análisis de las ideas sociológicas argentinas" (Echánove Trujillo, 1942, p. 255). 
aunque tanto en el cuerpo central como en la sección "Galería de sociólogos americanos", hay algunas menciones sobre las cátedras y profesores puntuales de esas instituciones. Vale recordar que el propio Ricardo Levene se desempeñó como profesor de Sociología en la FFyL de la UBA, y también en la Facultad de Humanidades y Ciencias de la Educación de la Universidad de La Plata.

Otro dato es que, tanto en el caso de Santa Fe como en el de Tucumán, quienes están al frente de las cátedras son exiliados: uno español, Ayala, y otro italiano, Treves. A su vez, como se observa en la subdivisión, figura el nombre de Jordán Bruno Genta, en Paraná, quien también se desempeñó como profesor adjunto de Alberto Baldrich, en Rosario — unidad académica que formaba parte en ese momento de la Universidad Nacional del Litoral (UNL) -; ambos fueron figuras sobresalientes del integrismo católico y quienes cumplirían un rol activo importante dentro del Golpe de Estado de 1943, en Argentina.

Para analizar este segmento del informe quisiéramos dividirlo en dos grupos. Por un lado, los casos de Córdoba, Paraná y Rosario, y, por otro, los de Tucumán y Santa Fe.

Dentro del primer grupo podemos observar que quienes están a cargo de las cátedras de Sociología en estos espacios universitarios — Raúl Orgaz y Alfredo Poviña, en la Facultad de Derecho y en el Instituto de Humanidades de la Universidad Nacional de Córdoba; Jordán Bruno Genta, en el Instituto Nacional del Profesorado en Paraná; Alfredo Baldrich, en la Facultad de Ciencias Económicas de la UNL en Rosario-, se orientan en mayor o menor medida a enseñar sociología.

En el caso cordobés esta enseñanza consiste en un recorrido desde una perspectiva histórica de las ideas sociales argentinas, en la que aparecen citados "el pensamiento sociológico de Echeverría, Alberdi, Sarmiento e Ingenieros" (Poviña, 1942, p. 257). Pero además se realizan "trabajos prácticos" sobre las obras de Vico, Montesquieu, Comte, Rousseau, Tarde, Durkheim, Stammler e 
Ingenieros. En su informe de presentación, Poviña agrega que se han dictado conferencias en el Instituto de Humanidades, una titulada "La conciencia social", a cargo de Orgaz, y otra sobre "La metodología sociológica de Max Weber", a cargo del propio Poviña, quien además agrega, para finalizar, que acababa de editarse en México su libro Historia de la Sociología en Latinoamérica.

Siguiendo con Jordán Bruno Genta, en su informe se observa un fuerte apego a la filosofía, así como al derecho y a una historia de corte institucional, todas las instancias tamizadas por una lente católica y con un encuadre desde lo político. Por otra parte, menciona que se ha publicado un libro de su autoría: Sociología política (1940) y que

[a]demás, el Instituto de Derecho público y Ciencias Sociales de Rosario, publicará próximamente un folleto con los dos primeros capítulos de un libro en preparación, La sociología y la política de Hegel, que constituirá un documento más sobre el contenido y orientación de la cátedra del Instituto del Profesorado de Paraná (Genta, 1942, p. 258).

Aquí podemos establecer una relación con Baldrich en Rosario (quien a su vez, junto con Poviña, eran profesores adjuntos de Levene en la UBA), así como también podemos prestar atención a la existencia de otro instituto de Sociología, esta vez en la UNL, “[p]aralelamente a la labor de la cátedra, se desarrollan las tareas del Instituto de Sociología, creado el año pasado y cuya tarea se presenta bajo tres aspectos: Conferencias, Reuniones y Publicaciones" (Baldrich, 1942, p. 259). En este informe, Baldrich detalla las tareas desarrolladas por el Instituto de Sociología de la UNL, exponiendo que las conferencias realizadas estuvieron a cargo de Diego L. Molinari ("La tradición nacional”) y de Alfredo Poviña ("Panorama de la sociología argentina"); además, menciona un acto académico, con varias intervenciones, incluida la del propio Baldrich, en "honor" a Mario Amadeo, representante del ministro de Relaciones Exteriores y Culto de la Nación. Sobre 
las "reuniones", da cuenta de los temas tratados: el sentido social en la obra La ciudad Indiana de Juan Agustín García; democracia política y económica; estudio del libro De Monarchia, de Dante, y el trabajo en la niñez. No hay mayores precisiones sobre quiénes participan de la organización del Instituto.

Esta búsqueda de institucionalización por fuera de la cátedra, en el caso de Rosario, pareciera más una extensión directa de la cátedra antes que un verdadero proyecto sociológico. Por otra parte, la perspectiva - poco desarrollada - muestra una lectura de la sociología como parte principal de la política, según el planteo de Baldrich, "tal como fue definida y estructurada en líneas esenciales por Aristóteles; continuada y perfeccionada por Santo Tomás y los grandes maestros de la escolástica moderna" (Baldrich, 1942, p. 259). Estas definiciones están en consonancia con lo que ya veníamos dilucidando de manera general respecto de la cátedra presentada por Genta en Paraná.

El segundo grupo está integrado por Tucumán y Santa Fe; este último será desarrollado en el apartado siguiente. Consideremos en esta parte el caso de la Universidad Nacional de Tucumán (UNT), a cargo de Renato Treves ${ }^{5}$.

Según Treves (1942),

los trabajos de sociología se han desarrollado preferentemente en torno a la cátedra de esta disciplina instituida en 1940 (...) y en la Sección de Sociología del Instituto de Investigaciones Económicas y Sociológicas, constitui-

5 Como mencionamos, la constitución de institutos está vinculada a las modificaciones introducidas desde la Reforma Universitaria, en tanto dispositivo modernizador de los espacios universitarios. Un caso poco estudiado es el Instituto de Investigaciones Económicas y Sociológicas (IIES) de la UNT, creado en 1940 —en un espacio universitario nacionalizado en 1921- como modo de pensar la planificación desde una perspectiva regional. El área de sociología fue encargada a un exiliado italiano que había arribado a Tucumán en 1939 luego de su paso por Montevideo y Buenos Aires: Renato Treves. Como asistente de la dirección se desempeñó Miguel Figueroa Román, quien se haría cargo del IIES en las etapas posteriores (Pereyra, 2012; Blanco, 2006). 
da también en 1940 y dependiente del Departamento de Investigaciones Regionales de la Universidad (p. 264).

En su informe, Treves da una explicación general de los puntos de su programa de cátedra, que se divide en dos partes: una introducción a la disciplina y otra que estudia las concepciones de la sociedad en la primera mitad del siglo XIX, de lo que se deduce que trabaja los "precursores" del pensamiento sociológico regional. También hace un resumido repaso sobre su última publicación Sociología y filosofía social, aparecida en la editorial Losada en 1941, cuyo apéndice sobre el pensamiento italiano fue publicado en la Revista Mexicana de Sociología ese mismo año.

Luego del repaso, el italiano pone el acento en la Sección de Sociología del Instituto de Investigaciones Económicas y Sociológicas (IIES), en donde "se han realizado con preferencia estudios de sociografía regional". Asimismo dice que "ha iniciado teóricamente estos estudios con un cursillo sobre los caracteres y los métodos de la sociología y sus relaciones con la sociografía". De manera paralela, Treves informa que Miguel Figueroa Román, en su función de consejero adscripto del IIES, brindó conferencias y presidió reuniones "con el fin de adiestrar un grupo de investigadores para el estudio de los problemas sociográficos de la provincia" (Treves, 1942, p. 264).

Para finalizar Treves informa que Figueroa Román había comenzado una investigación sobre "Sociografía de la clase obrera en Tucumán", junto a nutrido grupo compuesto por profesionales, estudiantes, dirigentes de asociaciones obreras, entre otros, que contó con el apoyo oficial por decreto del Poder Ejecutivo provincial, dada la incumbencia y los posibles aportes para sus fines gubernamentales.

Esta investigación se proponía en tres partes: en primera instancia se trabajaba con los "individuos", particularmente con los niños hijos de la clase obrera, a los cuales se los sometía a "investigaciones antropométricas" y de "desarrollo mental" de manera comparada con los de "clases acomodadas". Complementándose esta 
parte con estudios etnográficos, usos y costumbres, lenguaje, etc., a modo de fichar para crear una "memoria descriptiva" de los distintos puntos de la provincia tucumana. La segunda parte se refería "al medio", para lo cual se atendía "tanto el material (alimentación, habitación, vestidos, trabajo, salubridad, etc.), como el moral (educación, alcoholismo, diversiones, criminalidad, etc.)". La tercera parte comprendía la "economía familiar" postulándose el análisis del salario y el costo de vida, "con determinación de los números índices, salarios reales, etc.". La forma de obtener esta información era a través de la entrega de 3000 libretas a familias obreras, en las que se esperaba anotaran diariamente sus gastos, mientras que los gastos anuales se obtendrían mediante un cuestionario.

\section{El caso de Santa Fe y Francisco Ayala}

En la sección del informe general "Notas sobre la enseñanza de la sociología en América y la Argentina", Francisco Ayala presenta un breve estado sobre la cátedra de Sociología de la Facultad de Ciencias Jurídicas y Sociales de la UNL, en Santa Fe, en la cual se desempeñaba como profesor. Con una lógica distinguida, propia de la escritura ayaliana, no solo analiza el estado de la cuestión en particular sino que, a la par, deja algunas observaciones críticas de la disciplina en general.

Luego de realizar un rápido repaso casi genealógico de la cátedra santafesina de la carrera de Doctorado en Derecho, el autor realiza una exposición de los principales temas abordados en las unidades que componen su propuesta para el "Programa" de sociología, elevado al Consejo de la facultad para su aprobación.

Ayala parte de reconocer que la sociología es una "ciencia de constitución relativamente reciente" y,

[q]uizás por haberse formado en una hora tardía y crítica de nuestra cultura y en un medio agitado por las disensiones y contrastes más agudos aparece dividida en ramas nacionales separadas $y$, dentro de cada una, 
en corrientes y orientaciones casi siempre inconciliables (Ayala, 1942a, p. 262).

La sociología, siguiendo a Ayala, no es saber acumulativo en el que se puede "prescindir de la historia de la ciencia misma", sino que se presenta dividido en "sistemas independientes", "construcciones cerradas", en el que la aceptación de uno casi siempre implica la crítica y/o el rechazo de los otros. Esta particularidad de la disciplina se contrapondría con las ciencias matemáticas, físicas o biológicas, identificadas por el autor con las primeras caracterizaciones.

Por otra parte, mediante su planteo, Ayala argumenta la necesidad de una historia de la ciencia basándose en la divergencia con las otras ciencias y en el desarrollo propio de la sociología. Es así que, en la segunda parte del "Programa", luego de introducir en la primera parte el surgimiento y su ubicación en el campo de los "conocimientos humanos" y tratar de definir sus "sentidos",

el programa desarrolla un panorama histórico de esta ciencia, cuya finalidad es seguir el despliegue de la disciplina desde su iniciación como ciencia hasta el momento actual en aquellos medios culturales donde ha sido cultivada con rigor, continuidad y espíritu sistemático (Ayala, 1942a, p. 262).

Siguiendo el hilo implícito de esta argumentación, se podría decir que, para este autor, no hay sociología sin historia, solo en el devenir puede comprenderse el particular desarrollo, sus diferentes ramas o "sistemas" y su actual estadio. La historia de la ciencia entonces, en la interpretación de Ayala, se torna fundamental, dado que colabora mediante elementos, dimensiones y diálogos contextuales necesarios para observar y comprender cómo y por qué problemáticas situadas se tradujeron en cuerpos teóricos, que a su vez trataron de dar respuestas a esas problemáticas, es decir, una interacción específica y constitucional entre teoría y práctica, que puede iluminarse a partir de ubicar los parámetros en situaciones socio-históricas concretas. 
Pierre Bourdieu exponía que "la historia social de las ciencias sociales no es una especialidad entre otras. Es el instrumento privilegiado de la reflexividad crítica" (1999, p. 111). Siguiendo a Bourdieu, historiar el desarrollo de estas disciplinas descubre su fundamento cuando actualiza los presupuestos que se encuentran inscriptos en los propios principios fundantes de las empresas científicas; estos principios subsisten en el legado científico colectivo, en problemáticas, conceptos, métodos o técnicas. Desde esta perspectiva bourdiana podemos comprender la propuesta ayaliana, que postula la historia de la disciplina como una herramienta primordial.

Es este el instrumento que le permite introducir y comprender la sociología al granadino, así como su modernidad, pero también es uno de los sostenes por el que puede construir distinciones y demarcaciones con otros campos, en particular con el de las ciencias biológico-matemáticas. ¿Por qué expresa esta necesidad de establecer diferencias entre los campos en este momento? Sin duda que las tradiciones en las que se formó cobran una gran importancia en este punto, así como el contexto singular en el que está reflexionando, dado que la sociología en Argentina, si bien tiene una trayectoria institucional universitaria, esa trayectoria está limitada a la enseñanza y recién, con estos impulsos institucionales y grupales, comienza a definirse gradualmente la búsqueda de un estatuto científico, un estatuto propio que le otorgue legitimidad como ciencia, pero a su vez con la particularidad necesaria para diferenciarla del resto de ciencias concurrentes.

Esto repercute sobre la posibilidad de objetivar conocimientos, es decir, en el momento en que Ayala hace su análisis no estaría observando un "campo disciplinar" constituido con leyes de funcionamiento establecidas, instituciones específicas, con posiciones consolidadas (Bourdieu, 2000), sino que su lectura lo llevaría a plantarse frente a un campo en construcción, en el que tanto los circuitos como los circulantes no están expresamente definidos, por lo cual casi todas las argumentaciones y trabajos 
con pretensión sociológica tienen cierta necesidad de establecer o plantear explícitamente diferencias respecto a otras disciplinas, en particular a las más demarcadas o definidas del momento. Esto va a marcar el desarrollo y en parte la identidad misma de la sociología como ciencia en este contexto.

Ayala distingue aquellos datos e inserta en sus lecturas, sin explicitarlo, una específica "tradición sociológica". Como bien estudió y desarrolló Ribes Leiva (2005/2006; 2007), Ayala está formado en dos tradiciones sociológicas: por un lado la española, la que se nombra la inscripción tanto de su maestro Adolfo Posada como de José Ortega y Gasset; pero, por otro, hay una fuerte influencia de la tradición alemana, en la que el granadino, así como otros miembros de la generación española de la guerra, se ve atraído por la sociología historicista que practicaban Freyer, Oppenheimer, Alfred y Max Weber, Mannheim y otros. Es en consonancia con esta línea que Ayala entiende que todo lo social es histórico y se interpreta desde lo social mismo, en ello va implicada la sociología.

Por otro lado, la intervención de Ayala es la de un agente que no pertenece aún, al momento de escribir el informe sobre Santa $\mathrm{Fe}$, a las tradiciones intelectuales argentinas o, mejor dicho, no está formado en ellas. Su acervo reunido desde varios soportes -traducidos en prácticas concretas- apenas comenzaba a ingresar en la tradición sociológica universitaria argentina, que era dispersa y fragmentada, o sea que recién se comenzaba a distinguir de manera gradual. Para lo cual es importante considerar algunas características básicas de las tradiciones intelectuales y las condiciones sociales y políticas de la producción local de conocimiento.

El primer elemento que podemos notar es la condición periférica del espacio social local respecto de los centros de conocimiento, reconocimiento y, por ende, consagración ${ }^{6}$. Pero a esta

6 No se pretende interpretar aisladamente el espacio local, cuestión que iría en contra de lo que estamos planteando - dado que quedan demostradas las 
ubicación además hay que sumar otra condición: dicho espacio no se encuentra claramente delimitado, recortado en especialidades con instituciones y leyes igual de específicas; en todo caso, hay una mayor profesionalización de los espacios y los cuerpos, espoleada por una modernización capitalista acelerada de la sociedad y la consiguiente diferenciación general de funciones desde principios del siglo que tal modernización generaba (Sarlo y Altamirano, 1997, pp. 161-199)7. En este escenario, lo que tenemos en relación a Ayala es, en principio, un lector de la situación de la sociología en Argentina y la región, cuyo capital cultural no está inserto en las tradiciones y recorridos regionales, pero que, desde su trabajo intelectual, toma referencias de las tradiciones sociológicas constituidas (y en las que se constituyó) para "reingresar" en la tradición sociológica local en construcción.

Hablamos de "reingreso" desde la argumentación ofrecida por Ribes Leiva (2007), porque de una manera amplia hay un ingreso previo del autor en busca de lecturas, ideas, problemáticas, referencias, pero que hace a su vez devoluciones a través de sus primeros escritos, de sus traducciones, clases, conferencias, etc. Pero el elemento a tener en cuenta es que Ayala solo será considerado en la tradición local cuando los pares lo ubiquen a él y, principalmente, a su obra dentro de la tradición en construcción. Teniendo como referencia que el granadino está publicando y escribiendo sobre sociología desde una cátedra universitaria y

intencionalidades de intercambio y apertura que están manifestando mediante la constitución de redes de circuitos y circulantes los agentes intervinientes en las situaciones concretas-, sino establecer las diferencias posicionales respecto de otros espacios. Es esta ubicación (y condición al mismo tiempo) la que otorga particularidades "nacionales" en el "carácter bifronte del campo de las ciencias: ser a la vez un espacio social como los otros y a la vez un espacio de intercambio científico" (Martínez, 2007, p. 15).

7 Como lo plantean Altamirano y Sarlo (1997), en el ‘900 comienzan a vislumbrarse indicadores del surgimiento de un campo cultural, y con ello la creciente profesionalización y constitución de la carrera de escritor; elementos que posibilitan distinguir gradualmente un microcosmos recortado, desde el que se escindirá, a su vez, un campo intelectual moderno - que, junto a sus agentes, circuitos y circulantes, se constituyen en nuestro principal interés-. 
en una revista con pretensiones de especialización regional, podemos decir que además de los juicios valorativos de sus pares, Ayala ya había comenzado a ingresar y ser parte de la tradición sociológica universitaria. Es decir, se había transformado en un concurrente del espacio local, interviniendo (y poniendo en juego) desde y con su capital particular ${ }^{8}$.

Además, en este informe sobre Santa Fe, Francisco Ayala explaya algunas problemáticas presentes en la introducción de su "Programa" para la cátedra de sociología, expone los fundamentos desde los que encarar la enseñanza introductoria:

Habida cuenta de este conjunto de circunstancias, entiendo que un profesor no está autorizado para imponer a los alumnos, con prevalimiento de la cátedra que ejerce, criterios científicos fundados en sus personales preferencias. Su misión ha de consistir más bien en informarlos acerca de la historia y estado actual de las especulaciones sociológicas de tono y estructura científica, ofreciéndoles un panorama completo del desarrollo y de la problemática de la disciplina, de manera que puedan conocer los antecedentes de cada planteamiento de problemas y sepan referir a sus fuentes y localizar el sentido de las doctrinas o de las investigaciones con que sus lecturas los enfrenten. (Ayala, 1942a, p. 262).

La extensa cita presenta una posición de Ayala respecto de la enseñanza, pero también aparecen estrategias de enseñanza y aprendizaje que no implican una simple reproducción de sa-

8 No es indiferente que Ayala viene de Europa y que estuvo en Alemania formándose, esto puede sumar la apuesta del "beneficio" que implicaría, para un campo en formación y periférico, incorporar un agente externo con determinada trayectoria dentro de algunos espacios centrales o menos marginales. Por otra parte, en España la sociología tenía, al momento, un desarrollo similar que en la Argentina; en este contexto los miembros de la Generación de la Guerra eran quienes habían iniciado (o comenzaban a intentarlo) la introducción sistemática de la disciplina; en gran medida eran los agentes modernizadores de la sociología en España (Martínez, 2007; Ribes Leiva, 2007; Morales Martín, 2017). 
beres y / o conocimientos, sino que se fundamentan en la necesidad de que los estudiantes tengan un panorama lo más amplio posible, en el que los mismos estudiantes, con el mayor grado autonomía, sepan encontrar y referir los temas y problemáticas que enfrenten. Se podría decir que la propuesta está relacionada con crear posibilidades de producción de conocimiento desde la cátedra, así como reflexionar críticamente junto a los estudiantes. Nuestro autor está pensando en la enseñanza de la sociología, pero no lo hace desde la reproducción sino desde una nueva producción fundamentada, a modo de introducción general, con un recorrido que posibilita brindar herramientas de decodificación de un campo en construcción, de los distintos lenguajes y problemas de los "sistemas independientes". Es una introducción que no impone la sociología sino que dispone $a$ la sociología, debido a que permite conocer, reflexionar y elegir referencias.

Siguiendo con la exposición del informe, Ayala presenta la tercera parte de su "Programa", en la que expone el estudio de cuestiones relativas al método y al objeto, bajo el título "Los problemas generales de la sociología". Sobre este apartado, el autor solo expresa que se trata del espacio en donde pueden compararse los principios y sobre todo los planteos metodológicos de "los diversos sistemas".

La cuarta parte del Programa trata de los conceptos fundamentales de la sociología, y es trazada como principal para el autor, dado que permite plantear

de manera crítica la problemática actual, mostrando lo que hay de común y lo que hay de divergente en los distintos planteamientos, e introduciendo a los alumnos (...) en la zona viva de la ciencia, en el campo de la investigación y la especulación (Ayala, 1942a, p. 263).

El "Programa" concluye con una última sección referida a "Perspectivas de una sociología argentina", en el cual se fundamenta: 
El sentido de la Sociología, reconocido casi sin excepción desde su fundador, es el de servir para la vida humana al establecer las condiciones objetivas de la realidad social. Todo esfuerzo científico debe tomar como base y como meta a un tiempo mismo la realidad inmediata del medio dentro del cual se cumple (Ayala, 1942a, p. 263).

Para Ayala, "zona viva de la ciencia" ubicada en el terreno de lo local, vuelca la posibilidad de investigar "sobre los datos propio del país, con vista a sus problemas y mediante el empleo de métodos y técnicas adecuados a ellos" (Ayala, 1942a, p. 263).

De esta manera, la propuesta ayaliana es un punto de fuga respecto de casi todo lo que se venía planteando desde las cátedras de sociología en Argentina, e incluso en una buena parte de Latinoamérica, en el contexto de 1941. Desde su "Programa" propone a los estudiantes la posibilidad de lecturas, intercambios y herramientas para que analicen e investiguen "la realidad inmediata del medio", es decir, está brindando las condiciones básicas de posibilidad de construcción de conocimiento. Cuestión que se concreta en la creación de un grupo de investigación, espoleado desde y con la cátedra en ese mismo año lectivo (Escobar, 2011, pp. 71-90).

Como se puede observar, el segundo grupo (compuesto por el caso tucumano, con Treves y Figueroa Román a la cabeza, y el santafesino con Ayala) establece diferencias respecto del primer grupo, en el que incluimos a Córdoba, Paraná y Rosario. Estos últimos no tienen un planteo de la investigación como una fase importante en la sociología — de hecho, no se habla de posibilidades de investigación en sus informes-, aun a pesar de que hay diferencias internas entre los participantes de este grupo ya que Córdoba tiene un mayor anclaje en perspectivas más sociológicas 9 . Los otros dos casos, es decir, el de Paraná y Rosario, están

9 Pese a que en una exposición tan breve las influencias parecieran que se "concretan más bien a la historia y análisis de las ideas sociológicas argentinas" (Echánove Trujillo, 1942, p. 255). 
orientados en una lectura filosófica de la sociología - filosofía atravesada a su vez por la historia y la jurisprudencia-. Pero todos los casos de este grupo coinciden en una sociología orientada a la enseñanza y, aunque haya conferencias y publicaciones, ello pareciera tratar de reforzar esta orientación ${ }^{10}$.

El segundo grupo pone a la sociología en una posición radicalmente distinta, ya que habilita a desprenderse de la reproducción y posibilitar así producciones locales. En el caso tucumano esto es bien patente, a pesar de que aún puede leerse la diferenciación propia del contexto entre una sociología teórica y una aplicada. Es así que aparece la mención a la "sociografía" para la investigación de campo, de la cual se ocupa en forma casi exclusiva Figuera Román, ya que Treves pareciera apuntarse para los momentos de reflexión y enseñanza, aunque es sin dudas quien habilita las puertas para el trabajo de investigación.

Es significativo, como lo remarcamos en otros párrafos, que sean dos exiliados quienes, en cierta manera, de acuerdo con el informe que presenta el Boletín, estén a cargo de las cátedras y sobre todo de las acciones renovadoras que llevan adelante desde sus espacios. Renovación que no se cierra sobre sí mismos, sino que se abre sobre un grupo mayor, creando posibilidades de una institucionalización diferenciable de la ya establecida. Es posible que su relación con las tradiciones intelectuales locales, así como los espacios institucionales, no fuera lo suficientemente arraigada como para reproducirla, por lo cual sus trabajos comenzaron desde los inicios a introducir quiebres.

10 Para el caso cordobés, el trabajo señero de Juan Marsal argumenta que se puede hablar de una "escuela cordobesa de sociología", debido a que en la Facultad de Derecho de la Universidad Nacional de Córdoba "se produce en la materia una tradición pedagógica profesoral (...)" (Marsal, 1963, p. 126). Aunque no se puede dejar de señalar que la opinión de Marsal está atravesada por el influjo germaniano, que se tornaba como la experiencia de referencia - y en este caso dominante- en las interpretaciones del momento. 
Las experiencias concretas que presentamos nos muestran una cierta pluralidad de perspectivas y emprendimientos que hablan de una sociología en renovación. El marco en el que fuimos anclando y desarrollando lo expuesto, es decir, el Instituto de Sociología de la UBA y su Boletín, dan cuenta de un punto de inflexión en la disciplina, en la reorganización de una sociología que busca recortarse - mediante un grupo de agentes, prácticas e instituciones - en tanto ciencia social específica.

\section{La integración regional a través del Boletín del Instituto de Sociología}

Otro escrito que Francisco Ayala publicó en el primer número del Boletín del Instituto de Sociología fue "Sociología: teoría y técnica". Este artículo pauta una primera aceptación en estos nacientes circuitos asociados a la sociología dentro de Argentina y, también, en la región latinoamericana. El artículo fue incluido en el cuerpo central de la publicación, pero lo más sugestivo es que esta comunicación es una reseña bibliográfica. Cabe preguntarse por qué no se incluyó en la sección "Comentarios" del Boletín, espacio asignado para este ejercicio de escritura. Trataremos de esbozar algunas posibles respuestas a esta interpelación.

La primera es que publicar a Ayala en el cuerpo central es destacar su notabilidad junto al resto de autores que allí aparecen, demostrando que el granadino es un referente en el campo, o al menos alguien importante. La publicación no puede permitirse, en este primer número, publicarlo en los "Comentarios" como si fuera un iniciado o como una comunicación menor.

Pero también es muy importante el libro que reseña el autor, ni más ni menos que Sociología: Teoría y Técnica ${ }^{11}$ de otro exiliado español, José Medina Echavarría. Ayala escribe este texto casi en

11 La primera edición del mencionado libro es una co-edición entre el Fondo de Cultura de México y el Colegio de México del año 1941. 
simultáneo con la aparición del libro y es una de las primeras notas sobre el mismo en la región latinoamericana:

La ocasión de este Boletín me incita a dejar registrada en sus páginas la aparición de un libro que no vacilo en considerar desde ahora como un acontecimiento de relieve extraordinario dentro del campo de la producción sociológica en nuestro ambiente cultural, ni en pronosticarle una huella prolongada y profunda (Ayala, 1942b, p. 101).

Sin duda que la elección del libro reseñado guarda relación directa con los objetivos del Instituto, ya que se promociona una obra sociológica producida en Latinoamérica, cuyo carácter es de marcada renovación, y que, como tempranamente diagnostica Ayala, tendrá "una huella prolongada y profunda".

No es menor el hecho de que, entre las palabras de halago, homenaje y las recomendaciones de lectura del libro de Medina al posible público del Boletín, Ayala decida discurrir sobre un punto que objeta, considerando que "el autor reconocerá en ella un homenaje más fiel y digno que el elogio amistoso, tan fácil por lo demás cuando, como en este caso ocurre, cualquier ponderación estaría ampliamente justificada por la excepcional valía de la obra" (Ayala, 1942b, p. 102). Nuestro autor cuestiona algo que Medina también hace: el dualismo naturaleza-espíritu. Ambos coinciden en la necesidad de unidad de análisis antes que separación, pero, según Ayala, Medina recae, a pesar de oponerse en sus críticas, en el mencionado dualismo.

Con este comentario, Ayala ingresa en uno de los puntos importantes del libro de Medina ${ }^{12}$ que, como dicho comentario sugiere, se basa en desdibujar la distinción entre naturaleza y espíritu, propia del contexto alemán en la fuerte demarcación de

12 Ribes Leiva (2003) habla de la propuesta de Medina de desdibujar la falsa separación entre naturaleza y espíritu como una "segunda síntesis" de Sociología: teoría y técnica, dentro de cuatro síntesis fundamentales que contiene la obra desde su lectura. 
principios de siglo XX entre ciencias sociales o históricas ("del espíritu", tal como las denominó Dilthey) y ciencias naturales. El objeto de la sociología, es decir, el hombre en sociedad, demanda en la línea argumentativa de Medina examinar tanto lo simbólico como lo natural, por lo cual Medina aborda ambas cuestiones desde la comprensión interpretativa (verstehen weberiana) y la descripción durkheimiana —aunque comienza a prestar atención a la sociología norteamericana-, como un modo de unificar el dualismo. La búsqueda de dar forma a un sustento teórico para la sociología pareciera hacer inevitable el tratamiento de cuestiones y temas que rayan con lo filosófico.

Esta reseña y la crítica que implica dan muestra de un lazo particular entre ambos exiliados, de un conjunto de perspectivas y problemas en común. Por lo cual, al escribir acerca del libro de Medina, Francisco Ayala deja expresada una estela sobre alguien con quien compartió carreras, espacios españoles y con el que tuvo una fuerte relación intelectual; la que puede verificarse en una mutua y recíproca influencia, cimentada en una prolongada amistad. A modo de breve ilustración, señalamos que compartieron un recorrido intelectual que se cruzó de modo permanente desde sus formaciones a través de ámbitos académicos, influencias de los mismos profesores, un corpus de lecturas y autores semejantes, tertulias madrileñas, uno y otro viajaron a Alemania para complementar sus trayectorias formativas, fueron letrados de las Cortes de la República, compartieron problemáticas y temas de indagación y reflexión, inclusive coincidirán en años posteriores, a principios de la década del ‘50, en el exilio en Puerto Rico, donde Ayala llega por recomendación de Medina Echavarría (Ribes Leiva, 2007; Morales Martín, 2014; 2017).

Alberto Ribes Leiva (2007) ubica en relieve esta reciprocidad:

Hubo entre ambos un fluido intercambio de ideas. Además, la búsqueda y el aprendizaje en las mismas fuentes, es decir, la utilización de un similar "enfoque socioló- 
gico" extraído de prácticamente la misma tradición sociológica, supondrán que se den abundantes similitudes en las obras de ambos, aunque también son notables las diferencias (p. 105).

Es así que Medina "está presente en los textos de Ayala, y una lectura simultánea de los textos de uno y de otro pone de manifiesto coincidencias y discrepancias, pese a que apenas se citan mutuamente" (Ribes Leiva, 2007, p. 105).

A esta relación también se refiere Morales Martín cuando investiga el vínculo de Medina y el Instituto de Sociología de la UBA — espacio con el que mantuvo "visibles relaciones intelectuales"-: "su amigo de juventud y compañero de exilio, Francisco Ayala, se encargó en diferentes momentos de divulgar su obra (...)" (2013, p. 7). Esta divulgación viene acompañada de la búsqueda de un estatuto científico para la sociología, problemática en la que, en este contexto, Ayala comparte y coincide con Medina una vez más. Esto puede observarse cuando, en una reunión en el Instituto, el granadino advertía sobre la necesidad de un enfoque que unificara el estatuto teórico y la práctica:

La cuestión es fijar la relación entre la teoría y la práctica. La teoría es previa, se necesita el aparato conceptual, que por sí sólo no basta, el sentido de colaboración estriba en la unión de la teoría con la práctica. Uno solo puede hacer la teoría y formular un concepto, pero como la Sociología es una ciencia pragmática a la vez que especulativa, el sistema de conceptos debe ser controlado con la realidad: la investigación del dato empírico no puede ser labor individual, porque es una tarea muy amplia. De ahí, la necesidad - en primer término- de la colaboración (Reuniones del Instituto, 1943, p. 348).

Este comentario da cuenta de los intereses comunes y de cómo Ayala presenta y reingresa continuamente a su "compañero de exilio", aunque de manera no explícita, ya que no hay una cita di- 
recta ${ }^{13}$. Cabría inclusive la posibilidad de conjeturar, a modo de continuar con la relación Ayala-Medina desde otro ángulo, hasta qué punto la vinculación de Medina Echevarría con el Instituto de Sociología no estuvo mediada e impulsada por Ayala. Morales Martín (2014) propone que se constituye un importante corredor de ideas entre Argentina y México a partir de la fuerte relación establecida y continuada por estos dos intelectuales del exilio, lo que explica quizá la pronta y continua llegada del catálogo del Fondo de Cultura Económica (en el cual Medina fue de suma importancia como director de colección y traductor), así como incluso dar a conocer a un joven Gino Germani la obra y luego al propio Medina Echavarría: “es más que innegable el importante rol que jugó Ayala en este corredor de ideas argentino-mexicano al vincular personal e intelectualmente, en diversos momentos, a Medina Echavarría con este grupo de sociólogos" (Morales Martín, 2013, p. 8).

Al focalizar en las vinculaciones, afinidades e intercambios nos encontramos con una visión sobre la sociología regional que comienza a construirse cada vez más de manera compartida. La traza de una renovación sociológica en la región comenzaba a asentarse en prácticas circulantes, afinidades, constructos institucionales, tradiciones intelectuales, trayectos, búsqueda de legitimidades (disciplinares, institucionales, vinculares); todo un variado espectro de acciones y estructuras que nos ordenan en la observación de un interés consciente y creciente de generar un programa de modernización regional en la definición y el hacer sociológico. Este interés, a su vez, contribuye a acelerar la delimitación y profesionalización de un campo en vías de especialización. El Boletín del Instituto de Sociología de la UBA, en el periodo abordado, se constituyó en una de las herramientas que alojaron y propiciaron este interés.

13 Sin duda, aunque hay una búsqueda bastante similar en Ayala y Medina respecto del estatuto científico de la sociología, como lo mencionábamos mediante la cita de Ribes Leiva (2007), también son importantes las discrepancias en este caso respecto del lugar y definición de la teoría y la práctica en la unificación de ambas (Moya López, 2013; Morales Martín, 2017). 


\section{Referencias}

Ayala, F. (1942a). Santa Fe. Boletín del Instituto de Sociología, 1, 261-264. Buenos Aires, Imprenta y Casa Editora Coni.

Ayala, F. (1942b). Sociología: Teoría y técnica. Boletín del Instituto de Sociología, 1, 101-105. Buenos Aires, Imprenta y Casa Editora Coni.

Baldrich, A. (1942). Rosario. Boletín del Instituto de Sociología, 1, 259-261. Buenos Aires, Imprenta y Casa Editora Coni.

Blanco, A. (2006). Razón y modernidad. Buenos Aires: Siglo XXI.

Blanco, A. y Jackson, L. (2015). Sociología en el espejo. Argentina, Bernal: UNQ.

Bourdieu, P. (1999). Intelectuales, política y poder (Trad. Alicia Gutiérrez). Buenos Aires: EUDEBA.

Bourdieu, P. (2000). Los usos sociales de la ciencia (Trad. Horacio Pons). Buenos Aires: Nueva Visión.

Buchbinder, P. (2007). Historia de las universidades argentinas. Buenos Aires: Sudamericana.

Devoto, F. y Pagano, N. (2009). Historia de la historiografía argentina. Buenos Aires: Sudamericana.

Echánove Trujillo, C. (1942). América del Sur. Boletín del Instituto de Sociología, 1, 249-256. Buenos Aires, Imprenta y Casa Editora Coni.

Escobar, L. (2011). Francisco Ayala y la Universidad nacional del Litoral. Granada: Ed. Universidad de Granada-Fundación Francisco Ayala.

Escobar, L. (2016). La cátedra de sociología de la Facultad de Ciencias Jurídicas y Sociales. Gonzalo Sozzo (Dir.). Hacer Derecho, 185-203. Santa Fe: Ediciones UNL.

Genta, B. (1942). Paraná. Boletín del Instituto de Sociología, 1, 257-259. Buenos Aires, Imprenta y Casa Editora Coni.

González Bollo, H. (1999). El nacimiento de la sociología empírica en la Argentina. Buenos Aires: Dunken.

Levene, R. (1947). La cátedra y el Instituto de Sociología de la Facultad de Filosofía y Letras de Buenos Aires. Boletín del Instituto de Sociología, 5, 125-137. Buenos Aires, Imprenta y Casa Editora Coni. 
Marsal, J. (1963). La sociología en Argentina. Buenos Aires: Libros del Mirasol.

Martínez, A. T. (2007). Lecturas y lectores de Bourdieu en la Argentina. Prismas, Revista de historia intelectual, 11, 11-30.

Morales Martín, J. (2013). Entrecruzamientos en el Instituto de Sociología de la Universidad de Buenos Aires (1955-1965) y sus derivaciones: movilidad académica y Latin American Studies. Primeras Jornadas de Sociología, Universidad Nacional de Cuyo (Mendoza). Recuperado el 26 de enero 2016, de: http:/ /bdigital.uncu.edu.ar/ objetos_digitales/4881/moralesponmesa25.pdf

Morales Martín, J. (2014). Un corredor de ideas entre México y Argentina. El intercambio científico e intelectual entre los sociólogos del exilio español. Iberoamericana, 53, 29-48. Berlín, Instituto Ibero-Americano.

Morales Martín, J. (2017). José Medina Echavarría: vida y sociología. México: El Colegio de México.

Moya López, L. (2013). José Medina Echavarría y sociología como ciencia social concreta (1939-1980). México: El Colegio de México.

Neiburg, F. (1998). Los intelectuales y la invención del peronismo. Buenos Aires: Alianza.

Pereyra, D. (2007). Cincuenta años de la carrera de sociología de la UBA. Algunas notas contra-celebratorias para repensar la historia de la Sociología en la Argentina. Revista Argentina de Sociología, 5, 9, 153-159. Buenos Aires, CPS.

Pereyra, D. (2012). Sociología y planificación en el primer peronismo. El caso del Instituto de Sociografía y Planeación de Tucumán (1940-1957). Apuntes de Investigación, 21, 109-130. Buenos Aires, CECYP.

Poviña, A. (1941). Historia de la sociología en Latinoamérica. México: FCE.

Poviña, A. (1942). Córdoba. Boletín del Instituto de Sociología, 1, 256-257. Buenos Aires, Imprenta y Casa Editora Coni.

Poviña, A. (1943). Panorama de la Sociología Argentina. Revista de la Facultad de Ciencias Económicas, Comerciales y Políticas, II, 1, 151-165. Rosario, UNL.

Rawicz, D. (2012). Gino Germani: socialismo liberal y sociología científica. Revista Andamios, 9, 19, 235-257. México, UNAM. 
Reuniones del Instituto (1943). Boletín del Instituto de Sociología, 3 (1943). Buenos Aires, Imprenta y Casa Editora Coni.

Ribes Leiva, A. (2003). La sociología de José Medina Echavarría (19031977) en el centenario de su nacimiento: teoría sociológica, divulgación y sociología del desarrollo. Revista Española de Investigaciones Sociológicas, 102, 263-272. Madrid, CIS.

Ribes Leiva, A. (2005/2006). El enfoque y la tradición sociológica. Sociológica, 6, 107-136. Coruña, Universidade da Coruña.

Ribes Leiva, A. (2007). Paisajes del siglo XX. Sociología y literatura en Francisco Ayala. Madrid: Biblioteca Nueva.

Sarlo, B. y Altamirano, C. (1997). Ensayos argentinos. Buenos Aires: Ariel.

Treves, R. (1942). Tucumán. Boletín del Instituto de Sociología, 1, 264-266. Buenos Aires, Imprenta y Casa Editora Coni. 